\title{
Ambivalenzen der (De-)Aktivierung: Altwerden im flexiblen Kapitalismus
}

\author{
Silke van Dyk \\ Stephan Lessenich
}

\begin{abstract}
Seit der Rentenreform 1957 hatten alte Menschen in Deutschland eine Sonderrolle inne: Vergleichsweise gut versorgt wurden sie in den „wohlverdienten Ruhestand“ entlassen und von gesellschaftlichen Erwartungen weitgehend entbunden. Im Zuge der Rede von der drohenden „Überalterung“ der Gesellschaft und der Feststellung, dass die Alten selbst zugleich immer „jünger“ und gesünder werden, hat sich das Blatt zuletzt jedoch radikal gewendet: Als aktive Gesellschaftsmitglieder entdeckt, wird von Menschen im Ruhestandsalter neuerdings erwartet, dass auch sie ihren Beitrag zu einer erfolgreichen gesellschaftlichen Bewältigung der Herausforderungen des flexiblen Kapitalismus leisten. Den Ambivalenzen dieser Entwicklung geht der folgende Artikel nach.
\end{abstract}

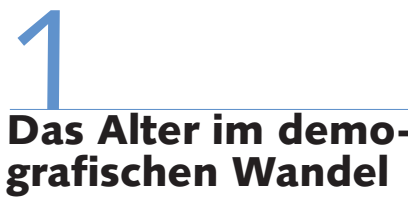

Im Zuge des Umbaus des Sozialstaats von einem „versorgenden“ zu einem „aktivierenden" Arrangement sozialer Unterstützung ist auch das Alter - die nachberufliche Lebensphase dauerhaften Transfereinkommensbezugs - zum Gegenstand gesellschaftlicher Neuverhandlung geworden. Die zunehmende Sensibilisierung der Öffentlichkeit für den doppelten Sachverhalt, dass „die Alten“ nicht nur „immer älter“, sondern in gewisser Weise auch „immer jünger" werden, also einen länger werdenden Teil ihrer länger werdenden Lebenszeit gesund und leistungsfähig bleiben, hat zur sozialpolitischen Entdeckung der Aktivierbarkeit des Alters geführt. Tonangebend hierfür ist hierzulande der Fünfte Altenbericht gewesen, mit dem das zuständige Bundesministerium einer hochkarätig besetzten Expertenkommission die Suche nach Antworten auf die Frage aufgetragen hatte, wie „die Potenziale des Alters gesellschaftlich besser genutzt werden können" (BMFSFJ 2005, S. 3). Die entsprechende Suche der Kommission nach „noch nicht verwirklichten Möglichkeiten“ älterer Menschen, „einen Beitrag zum Wohl der Solidargemeinschaft zu leisten" (ebd., S. 28), war dann - wenig überraschend - äußerst erfolgreich: Als gesellschaftlich noch ungenutzte oder jedenfalls besser nutzbare Potenziale des Alters wurden von ihr nämlich „neben materiellen Ressourcen insbesondere Gesundheit, Leistungsfähigkeit, Lernfähigkeit, Interesse, Zeit, Erfahrungen und Wissen" (ebd.) identifiziert. In entschie- dener Abgrenzung von überkommenen Bildern des greisen, siechen, hilfsbedürftigen Alters erscheinen die Älteren nunmehr geradezu als Quelle gesellschaftlich segensreicher Aktivität. Auch jenseits der Expertise der Altenberichtskommission und anderer wissenschaftlicher Zirkel (Erlinghagen/Hank 2008) hat sich dieses neue Bild des ,jungen“ Alters in jüngster Zeit auch in der Medienberichterstattung und nicht zuletzt in der Werbung zunehmend durchgesetzt.

Stellt man den politisch-ökonomischen Kontext des nunmehr zu Ende gehenden ersten Jahrzehnts des 21. Jahrhunderts in Rechnung, so erscheint die neue Rahmung des Alters als einer Phase fortgesetzter wenngleich anders gearteter, nachberuflicher - Aktivität alles andere als abwegig. Was läge in Zeiten chronisch beklagter fiskalischer Nöte der öffentlichen Hand, im Zeichen der prognostizierten (und fast schon reflexhaft als „Überalterung“ etikettierten) Zunahme des Anteils älterer Menschen an der Gesamtbevölkerung, im Windschatten schließlich der mit großem finanziellen, institutionellen und propagandistischen Aufwand betriebenen Aktivierung erwerbsfähiger Erwerbsloser näher, als auch „das Alter" wieder stärker in die gesellschaftliche Pflicht zu nehmen? Was klänge plausibler, als „die Produktivität älterer Menschen und die dem demografischen Wandel innewohnenden Chancen" (BMFSFJ 2005, S. 33) stärker in politische Planungen einzubeziehen? Wer könnte schon etwas gegen die Aktivierung der Alten, gegen die politische Belebung und gesellschaftliche Inanspruchnahme ihrer Lebensgeister haben oder sagen wollen? Und wer wollte wohl umgekehrt „auf den Vorzügen eines ,negativen', ,inkompeten- ten', ,unproduktiven', ,alten' Alters bestehen" (van Dyk 2007, S. 94)?

Der vorliegende Beitrag wagt einen kritischen Blick auf die Prozesse und Programme der Altersaktivierung. Er stellt sie in den Zusammenhang des gegenwärtig sich vollziehenden, sozialstaatlich vermittelten Übergangs zu einer neuen, aktivgesellschaftlichen Formation (Lessenich 2008a) und fragt nach den ihnen inhärenten Normalitätsannahmen, Widerspruchsmomenten und Konfliktpotenzialen.

\section{2 \\ Flexibler Kapitalismus und aktivierender Sozialstaat}

Die Rede von der „Aktivierung“ gibt seit nunmehr einem Jahrzehnt, spätestens aber seit den Sozialreformen rund um die "Agenda 2010“, in der bundesdeutschen Sozialpolitik den Ton an. Die Aktivierungsformel vom „Fördern und Fordern“, Leitlinie zunächst der mit dem Namen des damaligen VW-Personalvorstands Peter

\footnotetext{
Silke van Dyk, Dr., Wissenschaftliche Mitarbeiterin an der Friedrich-Schiller-Universität (FSU) Jena. Arbeitsschwerpunkte: Altersforschung, Wohlfahrtsstaatsforschung, Governmentality Studies, Diskurstheorie. e-mail: silke.vandyk@uni-jena.de Stephan Lessenich, Prof. Dr., lehrt Soziologie an der FSU Jena. Arbeitsschwerpunkte: Wohlfahrtsstaatsforschung, Altersforschung, Politische Soziologie, Zeitdiagnose. e-mail: stephan.lessenich@uni-jena.de
} 
Hartz verbundenen Arbeitsmarktreformen, hat sich mittlerweile auch in anderen Bereichen des deutschen Sozialstaats als regulative Idee sozialpolitischen Handelns durchgesetzt. Die im Bericht der „HartzKommission" formulierte politische Erwartungshaltung gegenüber im Sozialleistungsbezug stehenden Arbeitslosen, „den materiellen und nichtmateriellen Leistungen des Arbeitsamtes im Sinne der Schadensminderungspflicht durch ein angemessenes, zielführendes Verhalten zu begegnen" (Hartz et al. 2002, S. 45), richtet sich seither sinngemäß auch an andere Segmente der Sozialstaatsklientel: Unabhängig vom spezifischen Grund der aktuellen oder potenziellen Inanspruchnahme öffentlicher Leistungen werden sie in die Pflicht einer aktiven Mitwirkung an der Überwindung (oder Verhinderung) der sozialen Unterstützungssituation genommen.

Die neue Sozialpolitik der „Aktivierung" zielt in diesem Kontext auf die Ablösung jenes „Passivbürgers“, der, so die mittlerweile gängige Deutung, durch den expansiven „Versorgungsstaat" der langen Nachkriegszeit erschaffen worden wäre und dem ein Agieren im Sinne einer gesellschaftlichen Schadensminderungs- und -vermeidungspflicht zunehmend fremd geworden sei. Der idealtypische „Aktivbürger" hingegen bewegt sich, setzt sich ein und schaut voraus, mobilisiert seine Potenziale, befreit sich aus der Unmündigkeit, wird selbst tätig. Mit der Durchsetzung aktivierender Sozialpolitik nimmt das Prinzip selbstregulativer Prävention - ausgehend von der Arbeitsmarktpolitik und über den engeren Bereich gesundheitlicher Vor-Sorge hinaus - normativen Charakter an: Wer sich nicht frühzeitig und nachhaltig um sich selbst, um die Entwicklung seiner individuellen Fähig- und Fertigkeiten kümmert, wird zum Risiko für die Allgemeinheit stilisiert. Präventionspolitiken „laufen darauf hinaus, den Subjekten abzuverlangen, dass sie sich der Systemverträglichkeit ihres Handelns a priori vergewissern; dass sie systemzerstörende Effekte ihres eigenen Handelns präventiv ausschließen; dass sie sich als Risiko ernst nehmen und sich entsprechend selbst kontrollieren" (Vobruba 1983 , S. 40). So gesehen, etabliert der aktivierende Sozialstaat neben Zwangsmechanismen, die vor allem gegenüber Arbeitslosen zum Einsatz kommen, „ein Regime freiwilliger Selbstkontrolle" (Bröckling 2004, S. 214), das den Subjekten selbst auferlegt wird und tendenziell den gesamten
Lebenslauf der Sozialstaatsbürgerinnen und -bürger überformt - und mit dem sich entsprechend auch das höhere Lebensalter konfrontiert sieht.

\section{Das Alter(n) in der Aktivgesellschaft}

\subsection{AKTIVES ALTER(N) - EINE "WIN- WIN-SITUATION"?}

Die ersten Schritte der sozialstaatlichen Aktivierungspolitik in Deutschland gestalteten sich alles andere als konfliktfrei. An „Hartz IV“ entzündeten sich politische Auseinandersetzungen, die weit über den engeren Bereich der sozialpolitischen Kontroverse hinausgingen. Die Zusammenlegung von Arbeitslosen- und Sozialhilfe zum Zwecke der Mobilisierung erwerbsfähiger Erwerbsloser, eine Beschäftigung aufzunehmen, hat bei gewerkschaftsnahen Akteuren in Politik und Wissenschaft zu teils massiver Kritik an der „neuen Wohlfahrtsarchitektur“ (Urban 2004) des aktivierenden Sozialstaats geführt. Im Zentrum dieser Kritik stand die durch die Neugestaltung der Arbeitslosensicherung beförderte Tendenz zur „Rekommodifizierung " des Sozialleistungsrechts, sprich: der durch die Senkung von Leistungsniveaus und Bezugsdauern erhöhte „Zwang zur Verwertung der Arbeitskraft" (Bothfeld et al. 2004, S. 511).

Interessanterweise ist nun die Politik der Altersaktivierung, die sich parallel zum Umbau des Arbeitslosensicherungsregimes zu entfalten begonnen hat, zum Gegenstand der Kritik ebenfalls dort - und, so gilt es zu betonen, letztlich fast nur dort - geworden, wo auch sie sich als Akt der „Rekommodifizierung" deuten lässt. Insbesondere gilt dies mit Blick auf die erheblichen politischen Proteste, die die gesetzlich verordnete Verlängerung der Lebensarbeitszeit („Rente mit 67“) auf gewerkschaftlicher Seite hervorgerufen hat. Denn schließlich stellt diese Maßnahme zur Aktivierung älterer Arbeitnehmerinnen und Arbeitnehmer für abhängig Beschäftigte ebenfalls ganz offenkundig einen gegenüber der vorherigen Rechtslage erweiterten Zwang zum Verkauf der Arbeitskraft dar. In ähnlicher - wenngleich nach der politischen Durchsetzung ihrer betrieblichen Regulierung deutlich weniger massiven
Weise - wurde auch die „Riester-Rente“ bzw. die mit der Einführung der öffentlich geförderten privaten Altersvorsorge verkoppelte Absenkung des Alterssicherungsniveaus in der Gesetzlichen Rentenversicherung kritisiert. In beiden Fällen bezog sich die Problematisierung einer Programmatik der Altersaktivierung also im Kern auf die damit verbundene „Vermarktlichung" des Alters, d.h. auf die Marktnähe - und konkret: Arbeitsmarktnähe -, in die das Alter sozialpolitisch gerückt werde.

Gänzlich anders gelagert scheint hingegen die typische öffentliche - politische wie wissenschaftliche, nicht zuletzt auch dem „Arbeitnehmerlager“ zuzurechnende - Reaktion auf all jene Formen der Altersaktivierungspolitik zu sein, die auf Tätigkeiten älterer Menschen jenseits des Arbeitsmarkts zielen. Marktferne Betriebsamkeit im Alter, sei es nun im Sinne gesundheitsfördernder oder weiterbildender Aktivitäten, bürgerschaftlichen Engagements oder innerfamilialer Unterstützungsarbeit, wird im Grunde genommen von allen relevanten Akteuren des alterspolitischen Feldes als unbestreitbar wertvolles gesellschaftliches Gut propagiert und protegiert (Lessenich 2008a, S. 108ff.). Das viel-, ja einstimmige Lob der - nichtmarktförmigen - Aktivität im Alter entspringt einer wissenschaftlich-politischen Position, die im Kampf gegen das gesellschaftlich verbreitete Defizitbild des Alters praktisch jedwede Form des nacherwerblichen Tätigseins gutheißt - und für die umgekehrt jeder Zweifel an der Güte von Altersaktivierung quasi-automatisch in den Ruch der Altenfeindlichkeit geraten muss (van Dyk 2007, S. 97ff.). Dass bisweilen recht halbherzig - vor einer Instrumentalisierung der Altersaktivität gewarnt wird, tut diesem Tenor keinen Abbruch, wird diese Befürchtung doch kaum spezifiziert und die entsprechende Gefahr als durch "gute Politik“ vermeidbar eingestuft. Vollkommen offen bleibt dabei, nach welchen Kriterien der Punkt zu bestimmen wäre, an dem die emphatisch begrüßte, der Gesellschaft zugutekommende Nutzung der Altersaktivität in eine unbotmäßige gesellschaftliche Indienstnahme der Alten umschlagen würde.

Rund um das ,aktive Alter" und Politiken der Altersaktivierung hat sich auf diese Weise ein gesellschaftlicher Konsens gebildet, dem ,active ageing " als eine geradezu idealtypische „win-win-Situation“ gilt: 
„The beauty of this strategy is that it is good for everyone: from citizens of all ages as ageing individuals, in terms of maximizing their potential and quality of life, through to society as a whole, by getting the best from human capital, extending community participation and solidarity, avoiding intergenerational conflicts and creating a fairer, more inclusive society" (Walker 2002, S. 137). Das alternde Individuum, das sein Aktivitätspotenzial maximiert, erscheint hier rundum als ein Gewinn: für die Lebensqualität der Betroffenen selbst wie auch für das von der millionenfachen Nutzung dieses Potenzials profitierende gesellschaftliche Gemeinwesen. Eben diesen Tenor stimmt auch die Sachverständigenkommission zum Fünften Altenbericht der Bundesregierung an: „Aus individueller Perspektive ergeben sich im Vergleich zu früheren Generationen deutlich bessere Möglichkeiten, ein an eigenen Lebensentwürfen, Ziel- und Wertvorstellungen orientiertes Leben zu führen, an gesellschaftlicher Entwicklung teilzuhaben und sich für andere und die Gemeinschaft zu engagieren. Aus gesellschaftlicher Perspektive verweisen die Potenziale des Alters auf die Möglichkeit, durch motivationale, soziale, kulturelle und institutionelle Rahmenbedingungen dazu beizutragen, dass der wachsende Anteil älterer Menschen möglichst lange ein mitverantwortliches, selbstständiges und selbstverantwortliches Leben führt" (BMFSFJ 2005, S. 29).

Die Sachverständigen des aktiven Alters sind sich dabei einig, dass es hierfür nicht nur der förderlichen gesellschaftlichen Rahmensetzung bedarf, sondern vor allen Dingen auch der Einsicht der Subjekte in die Möglichkeit und Notwendigkeit von Altersaktivität: „Bei aller Betonung der sozialen und biografischen Voraussetzungen von Potenzialen des Alters darf nicht übersehen werden, dass Menschen bis ins sehr hohe Alter in der Lage sind, die Entwicklung entsprechender Potenziale durch eigenes Verhalten zu fördern“ (ebd., S. 31). Auch noch der alte Mensch ist also als tätiges, aktives, initiatives Wesen gefragt - als im aufgeklärten Eigen- wie Gemeininteresse agierender Unternehmer seiner „Alterskraft" (Lessenich 2008b). Und mehr noch: Es ist jeder Einzelne als „noch-nicht-altes“, aber unweigerlich alterndes, zukünftig altes Subjekt, der im wahrsten (nämlich den gesamten Lebenszyklus in den Blick nehmenden) Sinne des Wortes pro-aktiv Altersvorsorge betreiben soll: finanziell durch den Abschluss privater Zusatzversicherungen, gesundheitlich durch regelmäßige körperliche Betätigung vom Fitnesstraining bis zum Gehirnjogging - aber nicht zuletzt auch sozial: durch das vorsorgliche Knüpfen und Erhalten von Verwandtschafts-, Freundschafts- und Bekanntschaftsnetzen. Die Botschaft ist klar: Früh übt sich, wer auch im Alter noch aktiv sein will - und soll, denn, ,active ageing “ ist eben kein bloßes Privatvergnügen, sondern eine generationenübergreifende gesellschaftliche Aufgabe: „we are all part of the same project" (Walker 2002, S. 137).

\subsection{PROBLEME UND AMBIVALENZEN DER ALTERSAKTIVIERUNG}

„Zu schön, um wahr zu sein“ mag der eine oder andere Skeptiker ob dieser freudigen „win-win“-Botschaft denken. Tatsächlich ist die Skepsis berechtigt und hält auch einem zweiten kritischen Blick stand. Eines der beliebtesten Argumente der Altersaktivitäts-Apologeten ist der Hinweis darauf, dass mit der Aktivierung des Alters ein großer Schritt im Kampf gegen „Ageism“ und Altenfeindlichkeit vollzogen sei (z. B. Kleinemaß 2007), womit zugleich jegliche Aktivierungskritik im Verdacht einer eben solchen Abwertung des Alters steht. Auch wenn das Aktivitätspostulat durchaus positive Auswirkungen auf negative Alterskonnotationen wie Verfall und Passivität haben kann, blendet diese Perspektive gleichwohl aus, dass die Potenziale der Alten neuerdings vor allem deshalb interessieren, weil die Alten zugleich als Problem wahrgenommen werden. Es zeugt von einem offen negativen Altersbild, wenn der wachsende Anteil älterer Menschen an der Gesellschaft im Sinne einer „Überalterung“ als Last diskutiert wird, die jedoch von den ressourcenstarken aktiven Alten selbst kompensiert werden könne, angesichts der durch sie verursachten Belastung aber eben auch kompensiert werden solle.

\section{INDIVIDUELLE VERANTWORTUNGS- ÜBERTRAGUNG...}

So populär die Rede vom aktiven Alter ist, so konsequent wird zudem seine immer vorhandene Kehrseite - das pflegebedürftige, abhängige, passive und/oder demenziell erkrankte Alter - zu einer Fußnote der neuen Alterserzählung: Während junge Alte als (potenziell) aktive Subjekte adressiert werden, verbleiben hochaltrige Menschen im Objektstatus als zu Pflegende, zu Betreuende, zu Versorgende; man könnte von einem „Fahrstuhleffekt" ganz besonderer Art sprechen, wurden die klassischen Altersattribute doch einfach im Lebenslauf nach „oben“ verschoben: „[T]he ,new ageism ' [...] replaces an earlier generalized dread of ageing with a more specific fear of aging with a disability" (Holstein/Minkler 2003, S. 793). Zwar wird in der gerontologischen Diskussion von vielen Seiten auf die Gefahr hingewiesen, dass die Popularität des aktiven Alters durchaus zu einer Abwertung der Alten führen könne, die zur Aktivierung im gewünschten Sinne nicht mehr fähig sind (Lenz et al. 1999, S. 35). Diese Entwicklung wird jedoch als ebenso unerfreuliches wie vermeidbares „Beiprodukt" gehandelt und nicht als das, was sie tatsächlich ist: eine systematische Folge der zunehmenden Dominanz von Normen des mittleren Lebensalters. Tatsächlich dürfte sich dabei der Druck auf die heute Hochaltrigen im Vergleich zu früheren Generationen sogar erhöht haben, da mit der zunehmenden Infragestellung der biomedinizischen Defizitbestimmung, der Betonung der Plastizität des Alternsprozesses und der Popularisierung selbstregulativer Prävention das Nicht-Altern verstärkt zu einer Frage der (individuell zu verantwortenden) Entscheidung wird. Das hohe, abhängige, kranke Alter wird damit zum Scheiternsfall des „Unternehmen(s) Leben" (www.dak.de).

Neben der hier aufscheinenden inhärenten Abwertung des Alters treten weitere Probleme zutage, wenn die je individuellen Möglichkeiten, dem propagierten Aktivitätsideal zu entsprechen, in den Blick genommen werden. Denn den nach Geschlecht, sozialer Schicht- oder ethnischer Gruppenzugehörigkeit sehr unterschiedlich hohen Hürden auf dem Weg zur Annäherung an dieses Ideal wird bei der Feier des aktiven Alters üblicherweise kaum Rechnung getragen (kritisch: Backes 2006, S. 78ff.). Tatsächlich ist die Befähigung zur Selbstorganisation und zur permanenten (hoch kommerzialisierten) physischen und psychischen Selbstoptimierung jedoch in hohem Maße abhängig vom Bildungsstand und den finanziellen Ressourcen der Betroffenen. Auch die in der Debatte verhandelten, gewünschten öffentlichen Aktivitäten älterer Menschen haben einen starken „Mittelschichts-Bias“ - vom Nachhilfeunterricht für sozial schwache Kinder bis zur ehrenamtlichen Unterneh- 
mensberatung durch ehemalige leitende Angestellte. Die große Bedeutung der Sichtbarkeit des Alter(n)s wiederum ist eindeutig geschlechtsspezifisch strukturiert: Dass Altersmerkmale als „Verfallsmerkmale" gelesen werden, setzt bei Männern deutlich später ein als bei Frauen, obwohl Letztere durchschnittlich eine erheblich längere gesunde und aktive Lebensphase vor sich haben; vor längerer Zeit bereits sprach Susan Sontag (1972) deshalb von einem "double standard of ageing“. Angesichts dieser sozialstrukturellen Altersvielfalt muss immer wieder gefragt werden, inwiefern und in welchen Kontexten das propagierte Ideal des aktiven Alter(n)s am Alltag einer kleinen, privilegierten Minderheit orientiert ist, deren neu gewonnenen Freiheiten möglicherweise zu disziplinierenden Normierungen für andere werden.

Eine Problematisierung der Altersaktivierung kann sich aus unserer Sicht aber nicht auf die (deshalb nicht weniger bedeutsame) Frage beschränken, welchen (alten) Menschen qua Niedrigeinkommen, Geschlecht oder Pflegebedürftigkeit der Weg in die propagierte aktive Lebensführung versperrt ist. Auch das Ziel der Altersaktivierung selbst ist - jenseits der sozial beschränkten Zugangschancen - kritisch zu beleuchten. Nehmen wir diese Perspektive ein, offenbart sich ein weiteres zentrales Problem der Debatten um die Zukunft des Alter(n)s: Das neue Aktivitätsideal wird komplett losgelöst von den sozio-ökonomischen und -politischen Rahmenbedingungen diskutiert. Die Einbettung der Altersaktivierung in gesamtgesellschaftliche Dynamiken der Liberalisierung und Ökonomisierung bleibt trotz der auf der Hand liegenden Anschlüsse aus - ganz so, als würde die Altersaktivierung unter anderen Systembedingungen stattfinden als die Aktivierung jüngerer, erwerbsfähiger Menschen. Das aktive Alter wird damit im Jenseits des Kapitalismus und seiner aktuellen Transformationen verortet.

\section{...STATT GESELLSCHAFTLICHER VERANTWORTUNGSÜBERNAHME}

Hier rächt sich, dass die im angelsächsischen Raum einflussreiche Strömung der „Political Economy of Ageing“, die den Status des „Altseins“ als soziale Konstruktion unter kapitalistischen Vorzeichen analysiert (Estes 1979), im deutschsprachigen Raum bis heute kaum rezipiert worden ist.
Tatsächlich aber hilft eine theoretisch fundierte Rückbindung der Vergesellschaftung des Alter(n)s an ihre kapitalistischen Bedingungen, so manch naiven Kurzschluss zu vermeiden: So ist in einem gesellschaftlichen Kontext, in dem die Zeichen auf individuelle Verantwortungsübertragung stehen, in dem statt der sozialen Verhältnisse das Verhalten der Subjekte zum Ansatzpunkt politischer Steuerung geworden ist, realistischerweise nicht $\mathrm{zu}$ erwarten, dass die (nach wie vor altersfeindlich strukturierten) Rahmenbedingungen des Alter(n)s zum prominenten politischen Thema werden, wie es sich etwa die gerontologische Forschung (mehr oder weniger explizit) wünscht. Auch geht es im Zuge der Popularisierung von Altersaktivität eben weniger um den - von der Gerontologie betonten - individuellen Nutzen der Aktivität und das Wohlbefinden der adressierten Älteren als um den gesellschaftlichen Nutzen ihrer Lebensführung, die im Zeichen der Aktivgesellschaft auf die präventive Vermeidung und aktive Kompensation der potenziell durch das Alter entstehenden Belastungen zu zielen hat. Eingebettet in diesen Kontext wird also Altersaktivität als „Arbeit an sich selbst und an der eigenen Entwicklung" (Baltes/Montada 1996, S. 3) zum Wohle der Gesamtgesellschaft propagiert, während gleichzeitig bis dato gesellschaftlich gewährleistete soziale Sicherheiten im Feld der Renten-, Pflege- und Gesundheitspolitik reduziert werden, politische Initiativen gegen Altersdiskriminierung in Deutschland weiterhin in den Kinderschuhen stecken und es nach wie vor, aller demografiepolitischen Bewusstseinserweiterung zum Trotz, an altersgerechten Arbeitsplätzen fehlt. Anders als es Politikerinnen und Politiker sowie Gerontologinnen bzw. Gerontologen mehrheitlich tun, gilt es also nicht, vor der „bösen Instrumentalisierung" einer im Kern guten Altersaktivität zu warnen. Vielmehr geht es darum, den inhärent instrumentellen, das individuelle Wohlbefinden als Ressource einsetzenden Charakter des altersspezifischen Aktivitätspostulats zu benennen.

Dass die Aufforderung zur Altersaktivität einstweilen noch vor allem diskursiver Art ist, sollte nicht davon abhalten, diese gesellschaftspolitisch ernst zu nehmen und im Hinblick auf ihre Wirkungsmechanismen zu problematisieren. Denn es fällt auf, dass durch das neue Leitbild des aktiven Alter(n)s ,die gerade offensichtlich gewordene Pluralisierung des Alters [...] nun ei- ner, wenn man so will, neuen gesellschaftlichen Rahmung ausgesetzt (wird), deren Leitkriterien mit, gesellschaftlicher Verpflichtung' (...) und ,Remoralisierung umschrieben werden können" (von Kondratowitz 1998, S. 62). Hierbei geht es gerade nicht um die zwangsweise Verpflichtung zur Altersaktivität, sondern in erster Linie um die moralische Anleitung zum freiwillig-vernünftigen „Sich-Verhalten“ als alter(nder) Aktivbürger. Auch wenn empirisch zu überprüfen wäre, ob und inwiefern die gesellschaftliche Re-Normierung des Alter(n)s bei den adressierten Subjekten tatsächlich verfängt, sollte doch deutlich geworden sein, dass die Kritik an einer verpflichtenden Altersaktivität solange zu kurz greift, wie sie die gesellschaftliche und politische Anleitung zur eigeninitiativen Aktivität und Selbstsorge und den Preis für davon abweichendes Verhalten aus dem Blick verliert. Denn tatsächlich entsteht nicht nur für diejenigen ein Problem, denen es an den geeigneten Ressourcen fehlt, um dieser Aktivitätsanleitung Folge leisten zu können. Problematisch wird es auch für diejenigen, die einfach nicht mitmachen wollen und sich - obwohl sie über finanzielle, körperliche und mentale Ressourcen verfügen - den Aktivierungsanrufungen entziehen; sie mutieren damit zu pflichtvergessenen, verantwortungslosen Subjekten, denen ,eine Art von Wahnsinn“ - „der Unwille oder die Unfähigkeit, sich wie ein vernünftiges Subjekt zu verhalten" (Greco 2004, S. 296) - attestiert wird. In diesem Sinne ist jeder sozialpolitische Akt der Förderung von Engagement und Gesundheitsverhalten im Alter immer auch eine normierende "Zurichtung“ des Alters gemäß herrschender Aktivitätsmaßstäbe.

\subsection{NEOLIBERALE NORMIERUNG UND SELBSTBESTIMMTES ALTER(N)}

Zugleich würde es jedoch offensichtlich in die Irre führen, in diesem Prozess ausschließlich eine „neoliberale “Wende des Altersregimes zu sehen und die Impulse aus der kritischen Debatte um die Aktivierung der Erwerbsfähigen unvermittelt auf die Programmatik der Altersaktivierung zu übertragen. Die feministische Wohlfahrtsstaatsforschung (Knijn/Ostner 2002) hat dafür sensibilisiert, dass sozialpolitische De-Kommodifizierung nur für diejenigen ein soziales Recht auf (Markt-)Unabhängigkeit bedeutet, die unter den gegebenen 
gesellschaftlichen Bedingungen kommodifiziert sind. Und sie hat darauf hingewiesen, dass die Frage nach einer progressiven Sozialpolitik aus frauenpolitischer Perspektive neu formuliert werden muss. In analoger Weise müsste eine kritische Altersforschung aufzeigen, dass die Aktivierung von Menschen, deren erwerbsgesellschaftliche De-Aktivierung durch die Altersrente (nach wie vor) wohlfahrtsstaatliche Prämisse ist und deren Abwertung und Stereotypisierung wesentlich auf der festen Verankerung passivitätsorientierter Altersbilder beruht (z.B Filipp/Mayer 2005, S. 30), ebenfalls nach eigenen Maßstäben zu diskutieren ist und mindestens ambivalente Effekte produziert. Denn wenn bereits die Befähigung $\mathrm{zu}$ selbstbestimmtem und autonomem Handeln grundsätzlich infrage steht, wie dies bei älteren Menschen häufig und auch heute noch der Fall ist, bedürfen die Konsequenzen einer gesellschaftspolitischen Programmatik, die alte Menschen als KoProduzenten ihrer Lebensverhältnisse entdeckt und anerkennt, besonderer Aufmerksamkeit. So kritisch aus den skizzierten Gründen das Postulat des aktiven Alterns zu sehen ist, so wenig darf ausgeblendet werden, dass damit die tief verankerte, biomedizinisch begründete Defizitperspektive auf das Alter, die traditionell wenig Handlungsspielräume für alternde Menschen vorsah, zumindest erschüttert worden ist.

Jede Förderung von Autonomie und Eigenverantwortung, so instrumentell sie auch sein mag, birgt in sich ein subversives Potenzial, das zumal in einer gesellschaftlichen Gruppe, die strukturell arm an Anerkennungsressourcen ist, sukzessive gedeihen könnte. So müssen sich die von den aktivierten Alten gewählten Aktivitäten keineswegs mit den diskursiven Aktivitätsanrufungen im politischen und medialen Feld decken. Zu Recht weist Katz (2005, S. 131) darauf hin, dass sich in jüngerer Zeit zwar alle für die Aktivitäten der jungen Alten interessieren; niemand aber für deren "anti-activity activities", die in eigensinniger Weise den geforderten Ressourceninput zum Wohl der alternden Gesellschaft unterlaufen: Im Zweifelsfall könnte widerständischer Eigensinn dann sogar mit manisch-exzessivem Kreuzworträtsellösen oder der Zelebrierung des Kettenrauchens beginnen. Auch werden die jungen Alten, selbst wenn sie denn den an sie gerichteten Ansprüchen zunächst entsprechen und sich zu neuen Stützen des Sozialen auf- schwingen, irgendwann doch selbst auf Pflege angewiesen sein, ihre Ehrenämter niederlegen und nicht mehr jederzeit zur Enkelkinderbetreuung einspringen können. $\mathrm{Ob}$ sie sich dann still und genügsam aus der gesellschaftlichen Mitte verabschieden, um im Ohrensessel ihrer Großmütter und -väter Platz zu nehmen oder in schlecht ausgestatteten Pflegeheimen auf den Tod $\mathrm{zu}$ warten, wird seinerseits abzuwarten sein. Zu vermuten ist aber, dass „die (alten) Geister, die man rief", sich nicht ohne Weiteres wieder verabschieden werden. Ob, an welchem Punkt und für wen Ermächtigung in Eigensinn und Selbstbestimmung oder aber in Entmündigung und Unterwerfung umschlägt, ist dabei allein empirisch, wahrscheinlich selten eindeutig und nur vom Einzelfall ausgehend zu bestimmen. ${ }^{1}$

Um bei allen gegebenen individuellen und sozialen Unterschieden auch den gemeinsam geteilten Erfahrungen und Erfahrungsräumen alter Menschen nachgehen zu können, gilt es für die deutschsprachige Diskussion neben der ausgeprägten Fokussierung auf die wohlfahrtsstaatliche Versorgung des Alters Fragen der kulturellen und strukturellen Altersdiskriminierung stärker ins Zentrum der Aufmerksamkeit zu rücken. Bislang bleibt hierzulande unterthematisiert, dass und inwiefern alte Menschen im Kontext von wohlfahrtsstaatlichen Versorgungspolitiken immer auch stereotypisierend normiert und diszipliniert werden. Es fehlt an einer Perspektive, die es erlaubt, die normierende $\mathrm{Zu}$ richtung des Alters im Gesundheitssystem oder die Implikationen der zwangsweisen Ausgliederung aus dem Arbeitsmarkt kritisch zu diskutieren, ohne damit einer neoliberalen Wohlfahrtsstaatskritik, der Kürzung von sozialpolitischen Leistungsansprüchen älterer Menschen oder einer Rekommodifizierung des Alters das Wort zu reden. Wenn wir an dieser Stelle (auch) die verpflichtende Ruhestandsgrenze problematisieren, die in einer kapitalistischen Erwerbsgesellschaft mitsamt ihrer erwerbsarbeitsgestützten Anerkennungsordnung zu Statusverlusten für Rentnerinnen und Rentner führen kann, ist damit die grundlegende gesellschaftspolitische Frage aufgeworfen, wie Einkommen, Erwerbsarbeit, nicht-erwerbsförmige Tätigkeiten und der Raum für „Zweckfreies Sein“ im Kapitalismus so verteilt werden können, dass ein höchstmögliches Maß an Versorgung und Anerkennung für alle Alten, auch für die nicht oder nicht mehr „Leistungsfähigen“, garantiert ist.

Für ein tieferes Verständnis der altersspezifischen Aspekte der gesellschaftlichen Aktivierungsanrufung bleibt schließlich zu berücksichtigen, dass es auch die eigene Angst der Subjekte vor Alter und Tod ist, die als Motor für die Leugnung und Repression des Alters fungiert. Hazan (1994, S. 67f.) zeigt anschaulich, dass das gesamte gesellschaftliche Leben dadurch geprägt ist, dass Barrieren gegenüber dem Tod errichtet werden, sei es durch räumliche Ausgrenzung, durch Konversationstabus oder auch durch die sozialpolitische Abgrenzung und Konstituierung einer eigenständigen Altersphase. Die politische Propagierung eines aktiven Alters wäre vor diesem Hintergrund ein „Verdrängungsangebot“ an die im Zuge der Alterung der Gesellschaft immer größer werdende Gruppe Älterer, die angesichts steigender Lebenserwartung und verbesserter medizinischer Bedingungen nach Renteneintritt in der Regel noch 20 bis 30 Jahre vom Tod trennen. Das allgemeine Bestreben, aktiv und gesund zu sein, ist dabei sicherlich mehr als allein das Produkt eines liberalen Aktivierungsdiskurses und der Versprechen einer jugendorientierten „consumer culture“, bedienen doch beide zu unmittelbar den Impuls zur Verdrängung des Alters als letzte Station vor dem Tod. Umgekehrt scheint genau diese Verdrängung aber anschlussfähig an die Prämissen der Aktivgesellschaft zu sein: „Die subjektive Plausibilität des Verlangens, bis zu einem möglichst späten Tod ohne große Krankheiten und Beschwerden zu leben, korrespondiert (...) bezeichnend mit den Erwartungen der Außenwelt: des Arbeitsmarktes auf ungeschmälertes Leistungsvermögen, der Versicherungen auf einen kostenneutralen Lebensabend, der Gesellschaft insgesamt auf ein allzeit fittes, freundliches und optimistisches Erscheinungsbild“ (Siemons 2002).

\footnotetext{
1 Die Autoren des Artikels leiten das Forschungsprojekt "Vom ,verdienten Ruhestand“ zum ,Alterskraftunternehmer'? Bilder und Praktiken des Alter(n)s in der aktivgesellschaftlichen Transformation des deutschen Sozialstaats nach der Vereinigung ${ }^{\prime}$, in dem den hier aufgeworfenen Fragen empirisch im Ost-West-Vergleich nachgegangen wird (www.sfb580.uni-jena.de).
} 
Fazit

Altwerden im flexiblen Kapitalismus erweist sich in der Zusammenschau des Gesagten als hochambivalente Angelegenheit, der im Niemandsland von alternswissenschaftlicher, medialer und politischer Entproblematisierung des Aktivitätsideals einerseits, der weitgehenden Ausblendung der Alten in der Aktivierungsforschung andererseits bislang viel zu wenig kritische Aufmerksamkeit zuteil wird (vgl. als seltene Ausnahme z. B. Schroeter 2002). Weder kann und darf die sozialwissenschaftliche
Beobachtung der Altersaktivierung vom umfassenden Trend zur flexibilisierten Aktivgesellschaft entkoppelt werden noch ist es möglich, die Analysen der aktivierungskritischen Wohlfahrtsstaats- und Arbeitsmarktforschung einfach auf das Alter und dessen Aktivierung $\mathrm{zu}$ übertragen. Im Spannungsfeld von wachsenden Anerkennungs- und Gestaltungschancen für manche und zunehmender sozialer Entrechtung für viele, des allgemeinen Strebens nach einem schmerz- und beschwerdefreien Leben im Alter sowie der - wenn auch zunächst noch diskursiven - Inpflichtnahme der Alten für ein mögliches Scheitern im "Kampf mit dem Leben“ eröffnet sich ein relevantes Forschungsfeld.
Es lässt sich allerdings nur erschließen, wenn all diese Facetten zusammengebracht und -gedacht werden. Dabei kann zugleich nicht oft genug betont werden, dass die Kritik und Problematisierung der Altersaktivierung im flexiblen Kapitalismus nicht mit der rückwirkenden Legitimation der institutionalisierten Entmächtigung des Alters im „versorgenden “ Wohlfahrtsstaat verwechselt werden darf. Auch für die wohlfahrtsstaatliche Altenpolitik gilt, dass früher keineswegs „alles besser“ war. Der sozialwissenschaftlichen Kritik an der gegenwärtigen Entwicklung zum , aktivierenden" Sozialstaat muss diese Einsicht aber keinen Abbruch tun.

\section{LITERATUR}

Backes, G. (2006): Widersprüche und Ambivalenzen ehrenamtlicher und freiwilliger Arbeit im Alter, in: Schroeter, K. P./Zängl, P. (Hrsg.): Altern und bürgerschaftliches Engagement. Aspekte der Vergemeinschaftung und Vergesellschaftung in der Lebensphase Alter, Wiesbaden, S. 63-94 Baltes, M./Montada, L. (Hrsg.) (1996): Produktives Leben im Alter, Frankfurt/New York

Bundesministerium für Familie, Senioren, Frauen und Jugend (BMFSFJ) (2005): Fünfter Bericht zur Lage der älteren Generation in der Bundesrepublik Deutschland. Potenziale des Alters in Wirtschaft und Gesellschaft. Der Beitrag älterer Menschen zum Zusammenhalt der Generationen, Bericht der Sachverständigenkommission, Berlin

Bothfeld, S./Gronbach, S./Seibel, K. (2004): Eigenverantwortung in der Arbeitsmarktpolitik: Zwischen Handlungsautonomie und Zwangsmaßnahmen, in: WSI-Mitteilungen 4, S. 507-513

Bröckling, U. (2004): Prävention, in: Bröckling, U., et al. (Hrsg.): Glossar der Gegenwart, Frankfurt am Main, S. 210-215

van Dyk, S. (2007): Kompetent, aktiv, produktiv? Die Entdeckung der Alten in der Aktivgesellschaft, in: Prokla - Zeitschrift für kritische Sozialwissenschaft 146, S. 93-112

Erlinghagen, M./Hank, K. (Hrsg.) (2008): Produktives Altern und informelle Arbeit in modernen Gesellschaften. Theoretische und empirische Befunde, Wiesbaden

Estes, C. L. (1979): The Aging Enterprise, San Francisco

Filipp, S.-H./Mayer, A.-K. (2005): Zur Bedeutung von Altersstereotypen, in: Aus Politik und Zeitgeschichte B49-50, S. 25-30

Greco, M. (2004): Wellness, in: Bröckling, U., et al. (Hrsg.): Glossar der Gegenwart, Frankfurt am Main, S. 293-299
Hartz, P. et al. (2002): Moderne Dienstleistungen am Arbeitsmarkt. Vorschläge der Kommission zum Abbau der Arbeitslosigkeit und zur Umstrukturierung der Bundesanstalt für Arbeit, Berlin

Hazan, H. (1994): Old Age: Constructions and Deconstructions, Cambridge

Holstein, M./Minkler, M. (2003): Self, Society, and the "New Gerontology“, in: The Gerontologist 6, S. 487-496

Katz, S. (2005): Busy Bodies: Activity, Aging, and the Management of Everyday Life, in: Katz, S. (Hrsg.): Cultural Aging. Life Course, Lifestyle, and Senior Worlds, Peterborough, S. 121-139

Kleinemaß, U. (2007): Aktives Altern, Interview in: Das Apothekenmagazin der Deutschen Seniorenliga e. V., http://www.dsl-aktiv.com/ lebensart/altern/html (Zugriff: 13.08.2008)

Knijn, T./Ostner, I. (2002): Commodification and de-commodification, in: Hobson, B. et al. (Hrsg.): Contested Concepts in Gender and Social Politics, Cheltenham, S. 141-169

Kondratowitz, H.-J., von (1998): Vom gesellschaftlich „regulierten“ über das "unbestimmte" zum "disponiblen“ Alter, in: Clemens, W./Backes, G. (Hrsg.): Altern und Gesellschaft. Gesellschaftliche Modernisierung durch Altersstrukturwandel, Opladen, S. 61-82

Lenz, K./Rudolph, M./Sickendiek, U. (1999): Alter und Altern aus sozialgerontologischer Sicht, in: Lenz, K. et al. (Hrsg.): Die alternde Gesellschaft. Problemfelder gesellschaftlichen Umgangs mit Altern und Alter, Weinheim/München, S. 7-96

Lessenich, S. (2008a): Die Neuerfindung des Sozialen. Der Sozialstaat im flexiblen Kapitalismus, Bielefeld 
Lessenich, S. (2008b): Produktives Altern. Auf dem Weg zum Alterskraftunternehmer?, in: Füllsack, M. (Hrsg.): Verwerfungen moderner Arbeit. Zum Formwandel des Produktiven, Bielefeld, S. 45-64

Schroeter, K. R. (2002): Zur Allodoxie des „erfolgreichen“ und „produktiven Alter(n)s“, in: Backes, G. M./Clemens, W. (Hrsg.): Die Zukunft der Soziologie des Alter(n)s, Opladen, S. 85-110

Siemons, M. (2002): Werden Sie unsterblich. Nie wieder freie Radikale: Das neue Weltethos des Anti-Ageing, in: Frankfurter Allgemeine Zeitung vom 20.4., S. 41
Sontag, S. (1972): The double standard of aging, in: Saturday Review of Literature 39, S. 29-38

Urban, H.-J. (2004): Eigenverantwortung und Aktivierung - Stützpfeiler einer neuen Wohlfahrtsarchitektur?, in: WSI-Mitteilungen 9, S. 467-473 Vobruba, G. (1983): Prävention durch Selbstkontrolle, in: Wambach, M. M. (Hrsg.): Der Mensch als Risiko, Frankfurt am Main, S. 29-48 Walker, A. (2002): A strategy for active ageing, in: International Social Security Review 1, S. 121-139 